\title{
Report of an Unusual Case With Severe Fecal Impaction Responding to Medication Therapy
}

\author{
Wei Zhao, MD and Meiyun Ke, MD* \\ Department of Gastroenterology, PUMC Hospital, Chinese Academy of Medical Sciences, Peking Union Medical College, Beijing, China
}

Fecal impaction is a disorder characterized by a large mass of compacted feces in the rectum and/or colon, which cannot be evacuated. For mild and moderate fecal impaction, recommended treatments include stool softeners, oral mineral and olive oil, and edema; for severe fecal impaction, manual removal is needed and sometimes laparotomy may be indicated if medical therapies are not effective. Here we report a case with severe fecal impaction who did not defecate for 75 days. We treated this patient with vegetable oil, Chinese traditional medicine and enema in sequence. After 12 days of therapy, she evacuated hard fecal masses, and the symptoms were relieved.

(J Neurogastroenterol Motil 2010;16:199-202)

Key Words

Fecal impaction, Intestinal obstruction, Therapy

\section{Introduction}

Fecal impaction is a disorder characterized by a large mass of compacted feces in the rectum and/or colon, which cannot be evacuated. It is reported fecal impaction can occur in childhood, old age and some patients with spinal cord injury. ${ }^{1}$ With less systemic review, the true incidence is not certain. According to the location of the compacted feces and the degree of intestinal obstruction, the clinical manifestation varies, and treatments are affected depending on the extent, presence of megacolon, loss of colon innervation, and presence of other illnesses. Stool softeners, oral mineral and olive oil, and edema are valuable for mild and moderate fecal impaction. Severe fecal impaction or failure of these measures may necessitate surgery. Here we report a case of severe fecal impaction with no bowel movements for 75 days, in whom vegetable oil, Chinese traditional medicine and enema administered in sequence successively relieved the symptoms.

\section{Case Report}

A 55-year-old Chinese woman visited gastrointestinal clinic because of constipation for 3 months, characterized by lack of urge to defecate, hard stool, and infrequent stools (less than 1 time in 10 days). The symptoms were first manageable with but soon resistant to saline enema and lubricant laxative. Till being admitted to clinic, she did not have bowel moments for 61 days, with abdominal distention, abdominal pain, vomiting, and low-grade fever. Abdominal radiographs (Fig. 1) and computed tomography scan (Fig. 2) revealed a dilated colon filled with

Received: March 5th, 2010 Accepted: April 5th, 2010

(c) This is an Open Access article distributed under the terms of the Creative Commons Attribution Non-Commercial License (http://creativecommons. org/licenses/by-nc/3.0) which permits unrestricted non-commercial use, distribution, and reproduction in any medium, provided the original work is properly cited.

*Correspondence: Meiyun Ke, MD

Department of Gastroenterology, PUMC Hospital, Chinese Academy of Medical Sciences, Peking Union Medical College, Beijing 100730, China

Financial support: None.

Tel: +86-10-6526-5006, Fax: +86-10-6529-5052, E-mail: kemypumch2006@yahoo.com.cn

Conflicts of interest: None. 
feces. Barium enema, colonoscopy (performed twice, and once reaching the ileocecal junction) in local hospital showed abundant feces and fecal impactions. Routine blood test showed a lowered neutrophil level $\left(2,870\right.$ cells $/ \mu \mathrm{L}$ or $2.87 \times 10^{9}$ cells/L). Thyroid function tests, serum potassium, blood calcium and tumor markers were all normal. During this period, she underwent saline enema for more than 30 times. Fasting, gastrointestinal decom-

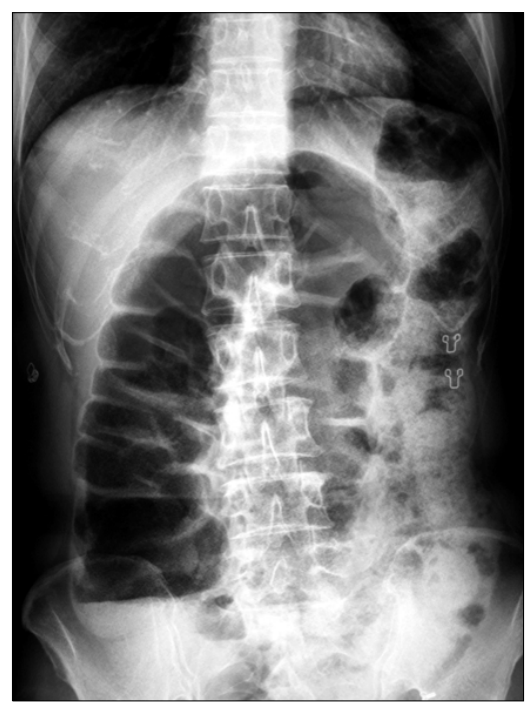

Figure 1. Abdominal radiograph. It shows a dilated colon with air-fluid level in the cecum, indicating the site of the intestinal obstruction. pression, intravenous broad-spectrum antibiotics, and nutrition support were also administered, but she still had abdominal pain, fullness, and failed to defecate.

Abdominal examination revealed several large, well palpable, hard abdominal masses, in the left and right lower quadrants, and hyperactive bowel sounds. Digital rectal examination showed an empty rectum and no anal fissures, stricture, or tenderness. Other examinations were negative. Follow-up andominal radiographs after barium enema showed abundant barium remained in the colon (Fig. 3).

The patient hesitated to receive operation. Two weeks later,

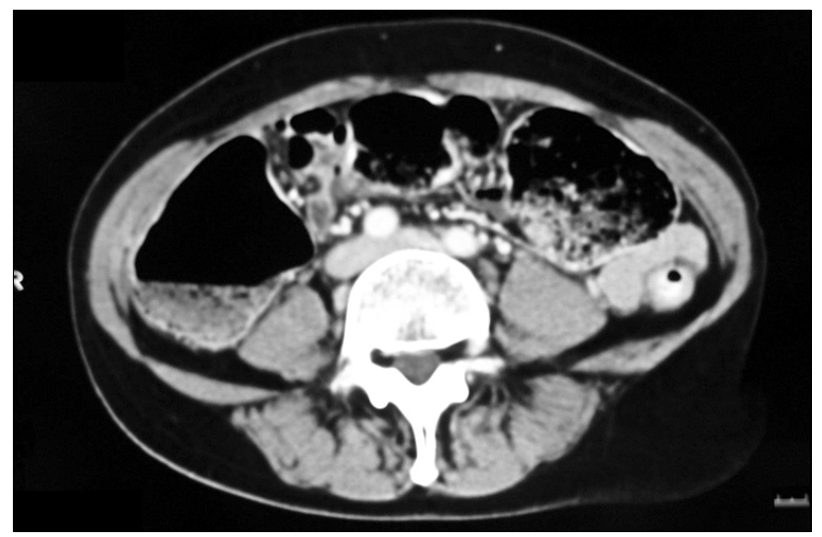

Figure 2. Arterial phase of abdominal computed tomography (CT) scan. It shows the dilated ascending and transverse colon without colon wall thickening. The lumen of splenic flexure is filled with abundant fecal materials.
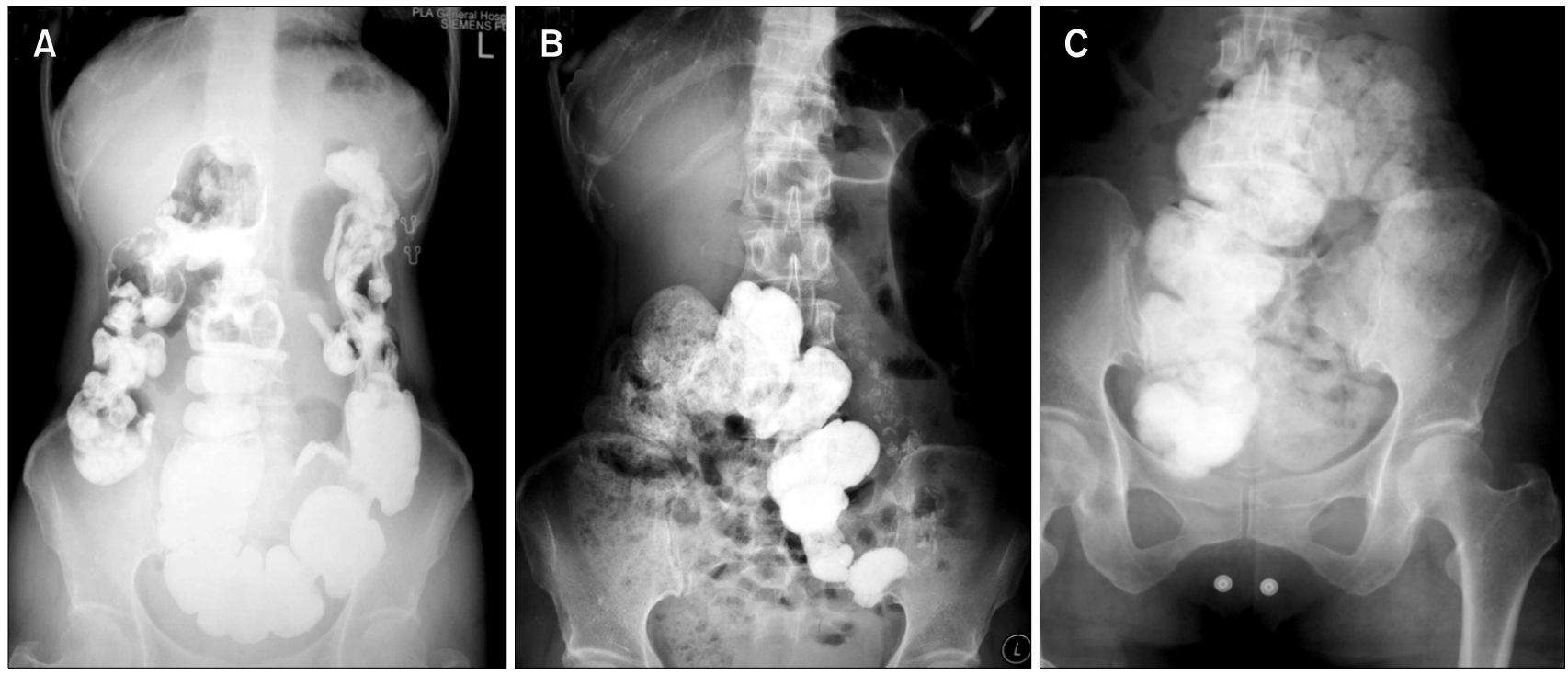

Figure 3. Follow-up abdominal radiographs after barium enema. Abundant barium remain in the 17th day (A), 24th day (B), and 33rd day (C). The fecal stasis is so obvious that it span from the cecum to the transverse colon, where it is well identified on the right of the abdomen. 
she visited gastrointestinal clinic again, and she occasionally expelled gas from the rectum. At first she was recommended to take water at small amounts several times per day $(800 \mathrm{~mL} /$ day $)$. Two to 3 days later, oral peanut oil was added (20-30 mL/time, 40-60 $\mathrm{mL} /$ day; gradually increased to $70-120 \mathrm{~mL} /$ day). The abdominal pain was not aggravated and there was still gas evacuation. Then Maren Ruanjiaonang ${ }^{\circledR}$ (Tianjin Central Pharm. Co. Ltd., 1 Fujin Road, Beichen square, Tianjin, China), a kind of Chinese traditional medicine, was added (2 capsules/time, 6-12 capsules/day) as an oral lubricant laxative. During the period, the patient vomited, and abdominal pain turned severer occasionally, and abdominal examination showed the displacement of some abdominal masses with negative peritoneal irritation sign. Five days later, saline enema in knee-chest position was administered, at the same time, volume of water intake was increased gradually to $1,000 \mathrm{~mL} /$ day. And the abdominal masses kept on moving to the left lower quadrant. At the 12th day of treatment, several pieces of hard stool were evacuated, and abdominal mass in the left lower quadrant turned smaller. After 87 days, the patient evacuated hard stool, and began to take liquid diet, supplied with enteral nutrition instead of parenteral nutrition. Over the next 6 months, the patient took Macrogol 4,000 (10-30 g/day), probiotics and prokinetic agent, together with enema, once every 2 weeks and she had bowel moment every day. She gains $15 \mathrm{~kg}$ in weight. Now the patient is still under follow- up.

\section{Discussion}

Fecal impaction can be found in every age, especially in childhood and old age. A study of geriatric wards in the United Kingdom showed that up to $42 \%$ of the old patients had fecal impaction. ${ }^{2}$ In some cases, it causes a serious situation; impaction of hard fecal mass on the colonic wall may cause stercoral ulcer, lower gastrointestinal bleeding, ${ }^{3}$ intussusception, ${ }^{4}$ or even perforation. ${ }^{5}$ For patients with fecal impactions, the aims of management are to relieve symptoms, clear out colon, and restore normal bowel habit. The most important regimen is to evacuate feces out of colon as soon as possible. Till now, research-based treatment of fecal impaction is very limited, and non-research based treatment varies. A study involved 10 patients with fecal impaction reported that osmotic laxative is effective for patients with no evidence of bowel obstruction. ${ }^{6}$ For those with rectal fecal impaction, Bekkali et $\mathrm{al}^{7}$ in their randomized controlled trial study ( $n=90)$, reported that enema and polyethylene glycol are equally effective and should be the first line therapy (successful dis- impaction rate was $80 \%$ achieved with enemas alone vs. $68 \%$ with polyethylene glycol alone, $\mathrm{p}=0.28$ ). The pulsed irrigation enhanced evacuation may also be effective. ${ }^{8}$ But cases with evidence of obstruction or being refractory to medication therapy need operation. It has not been reported that a fecal impaction with no defecation as long as 75 days and failed to respond to medication can also be relieved by nonsurgical therapy.

Successful therapy in this case focused on keeping mechanical balance of colon, to clear fecal impaction gradually. For bowel obstruction was present, food and water intake should be ceased, or else intra-colonic pressure would keep on increasing, which may develop complication of fecal perforation. There were multiple fecal impactions from the right to left part of colon in this case, and also the patient failed to respond well to multiple intestinal lavages in the past 3 months. Following fasting and parenteral nutrition, her abdominal pain and bloating were relieved. When there was some flatulence, the patient was asked to take a little water (she used to vomit after drinking), and the water volume increased gradually. Then vegetable oil was added, which was gradually combined with Chinese traditional medicine, hoping to soften and move hard fecal masses to the distal part of colon. With the moving of feces to the distal part of colon, saline enema was added to further soften colon contents and facilitate defecation directly, and, to reach, as far as possible, to the proximal part of rectum, in a knee-chest position. It should be emphasized that the parenteral nutrition made it possible for the patient to survive so long period of fecal impaction, and the cooperation between the patient, her family and the doctor was also very important.

Once fecal impaction is relieved, it is necessary to receive maintenance therapy to avoid recurrence. Periodic enema may be required for some patients in long-term care.' Radiographic study may be necessary to monitor the adequacy of treatment, especially in patients with recurrent fecal impaction. ${ }^{10}$ When fecal impaction is present, a limited enema study with a water-soluble contrast agent can outline the colon and fecal mass and rule out other diseases without aggravating the condition. But barium contrast should be used with caution. Otherwise, it could aggravate the situation, ${ }^{11}$ which could also be seen in this case.

As for the etiological factors of fecal impaction, mechanical obstruction, metabolic and endocrine disorders, medications, neurologic and myopathic disorders, and functional constipation should be considered. This patient, lacking clues for any organic disease and history of certain medication use, might have functional constipation. Furthermore, the patient's history, most like- 
ly represents slow-transit constipation. To confirm it, gastrointestinal transit time test would be performed later.

\section{References}

1. Read NW, Celik AF, Katsinelos P. Constipation and incontinence in the elderly. J Clin Gastroenterol 1995;20:61-70.

2. Madan P, Bhayana S, Chandra P, Hughes JI. Lower gastrointestinal bleeding: association with Sevelamer use. World J Gastroenterol 2008;14:2615-2616.

3. Mahajna A, Krausz MM. Ileocolic intussusception in an adult patient due to a cecal fecalith. Isr Med Assoc J 2009;11:58-59.

4. Arana-Arri E, Cortés H, Cabriada V, Lekerika N, García-Verdugo A, Shengelia-Shapiro L. Giant faecaloma causing perforation of the rectum presented as a subcutaneous emphysema, pneumoperitoneum and pneumomediastinum: a case report. Eur J Emerg Med 2007;14: 351-353.

5. Creason N, Sparks D. Fecal impaction: a review. Nurs Diagn 2000;1:15-23.
6. Tiongco FP, Tsang TK, Pollack J. Use of oral GoLytely solution in relief of refractory fecal impaction. Dig Dis Sci 1997;42:1454-1457.

7. Bekkali NL, van den Berg MM, Dijkgraaf MG, et al. Rectal fecal impaction treatment in childhood constipation: enemas versus high doses oral PEG. Pediatrics 2009;124:e1108-e1115.

8. Puet TA, Phen L, Hurst DL. Pulsed irrigation enhanced evacuation: new method for treating fecal impaction. Arch Phys Med Rehabil 1991;72:935-936.

9. Brocklehurst JC, Kirkland JL, Martin J, Ashford J. Constipation in long-stay elderly patients: its treatment and prevention by lactulose, poloxalkol-dihydroxyanthroquinolone and phosphate enemas. Gerontology 1983;29:181-184.

10. Gau JT, Acharya U, Marx T, Verne GN, Carlsen W. Megacolon and stercoral proctitis after frequent fecal impactions: report of an unusual case and review of the literature. J Am Med Dir Assoc 2007;8:338-341.

11. Claffey KB, Patton ML, Haith LR Jr, Germain TJ, Kerstein MD. Barium and fecal impaction: an unusual case of bilateral hydronephrosis. Am Surg 1995;61:709-713. 